\title{
Analysis of Expression Levels and Functions of MDK and PTN Genes in Colorectal Cancer
}

\author{
Fengqin Jiang \\ Huzhou Central Hospital \\ Huilian Huang \\ Huzhou Central Hospital \\ Yang Liu ( $\square$ liuyang2017@zju.edu.cn ) \\ Huzhou Central Hospital https://orcid.org/0000-0001-8528-1355
}

\section{Research Article}

Keywords: MDK, PTN, colorectal cancer, biomarker, immune infiltration

Posted Date: February 22nd, 2022

DOI: https://doi.org/10.21203/rs.3.rs-1314782/v1

License: (1) This work is licensed under a Creative Commons Attribution 4.0 International License. Read Full License 


\section{Abstract}

Purpose Colorectal cancer (CRC) is the third most common and the fourth most lethal type of cancer. Current therapies for CRC mainly include chemotherapy, radiotherapy, and surgery. However, the therapeutic effects of these therapies are not satisfactory for advanced CRC patients. Therefore, there has been ongoing research looking for better diagnostic targets that may give rise to more efficient interventions. Midkine (MDK) and pleiotrophin (PTN) are two important heparin-binding cytokines. These proteins are highly expressed in many human tumor cells, but have low or no expression in normal tissues.

Methods To identify novel diagnostic targets for CRC, we herein analyzed the expression patterns of $M D K$ and PTN genes in colorectal cancer and normal samples by using The Cancer Genome Atlas (TCGA) and tumor immune estimation resource (TIMER) databases.

Results We found that both genes were abnormal expressed in CRC samples and involved in the regulation of immune response and cell metabolism, and the expression levels of these two genes had significant prognostic value.

Conclusions In conclusion, our results provide a comprehensive understanding of the functions of MDK and PTN genes in colorectal cancer.

\section{Introduction}

Colorectal cancer (CRC) is the third most common and the fourth most lethal type of cancer [1-3]. During early stages, CRC can be effectively managed via surgical resections. In fact, patients with early-stage CRC have a long survival period and can even be completely cured. Data show that the 5-year survival rate of patients with stage I CRC is higher than $85 \%$ [4]. However, for patients with stage IV CRC, $65 \%$ may experience recurrence after surgical resections [5] and most of them exhibit disease progression due to resistance to chemotherapies and targeted drugs [6]. As a result, the 5-year survival rate of patients with stage IV CRC is less than 10\% [4]. Therefore, exploring novel targets for adjuvant therapy for CRC has become more and more important.

Midkine (MDK) and pleiotrophin (PTN) are two members of the heparin-binding cytokines family. The amino acid sequences of mammalian MDKs show $50 \%$ homology to those of mammalian PTNs $[7,8]$. In humans, MDK and PTN are abnormally expressed in many kinds of cancer cells [9], such as neuroblastoma [10], bladder cancer [11], lung cancer [12,13], breast cancer $[14,15]$ and thyroid papillary carcinoma $[16,17]$. In addition, studies have shown that both MDK and PTN are abnormally expressed in many inflammatory diseases [18]. In a previous study, we found that the $M D K$ gene was highly expressed in the CRC tissue relative to the normal tissue by immunohistology analysis, and the expression of $M D K$ was closely related to the clinical stage and degree of malignancy of CRC. This finding was consistent with those of some other studies, which indicated that $M D K$ was abnormally expressed in CRC; in addition, according to these studies, PTN was also expressed abnormally in CRC $[19,20]$. However, 
according to existing reports, the expression patterns of $M D K$ and PTN in CRC remain controversial. To clarify the expression patterns of these two genes and unveil their underlying regulatory mechanisms, in this study, by comprehensively analyzing the RNA-seq data on MDK and PTN genes, we explored the expression characteristics and potential biological functions of the two genes in CRC. The findings were then combined with the clinical characteristics of CRC patients to guide the establishment of new prognostic markers for CRC.

\section{Materials And Methods}

\section{Retrieval and processing of mRNA expression data}

We collected RNA-seq profiles (level 3 HTSeq-FPKM format) from the CRC cohort deposited in The Cancer Genome Atlas (TCGA) (https://portal.gdc.cancer.gov/), which contains 454 tumor samples and 41 normal samples. Then the FPKM (Fragments Per Kilobase per Million reads) data were converted into the TPM (Transcripts Per Million reads) format and log2-transformed. For data filtering, the clinical information was retained and duplicate samples were removed. $R$ (version 3.6.3) was used for statistical analysis of data, and the "ggplot2" (version 3.3.3) package in R was used for data visualization.

\section{Characterization of the expression levels of MDK and PTN genes}

Differences in mRNA expression levels of $M D K$ and PTN genes between the CRC and normal samples of the TCGA cohort were identified by t-test. R (version 3.6.3) was used for statistical analysis of data, and the "ggplot2" (version 3.3.3) package in R was used for data visualization.

\section{Gene set enrichment analysis (GSEA) of MDK and PTN genes}

The LinkedOmics database (http://www.linkedomics.org/) [21] was used for GSEA. Genes significantly correlated with the expression of $M D K$ and PTN were filtered by Pearson correlation analysis using the "LinkFinder" module of LinkedOmics. Then, Gene Ontology (GO) and Kyoto Encyclopedia of Genes and Genomes (KEGG) enrichment analyses were performed for the selected positively or negatively correlated genes by using the "LinkInterpreter" module of LinkedOmics.

\section{Analysis of the correlation between MDK and PTN expression levels and immune infiltration}

The tumor immune estimation resource (TIMER; https://cistrome.shinyapps.io/timer/) [22] database was used to analyze the correlation between $M D K$ and PTN expression and the levels of infiltrating immune cells, such as dendritic cells, CD4+ T cells, neutrophils, CD8+ T cells, macrophages, and B cells. The stromal score (that captures the presence of stroma in tumor tissue), immune score (that represents the infiltration of immune cells in tumor tissue), and ESTIMATE (Estimation of STromal and Immune cells in MAlignant Tumor tissues using Expression data) score[23] of each CRC patient were also analyzed by corresponding $\mathrm{R}$ packages to determine the infiltration levels of immune cells and stromal cells in CRC tissues [24]. Statistical analysis was performed by adopting Pearson's correlation coefficients. 


\section{Survival analysis of MDK and PTN expression levels}

Overall survival analysis was performed by R (3.6.3). The "survival" package (version 3.2.10) in R was used for statistical analysis of survival data, while the "surviviner" package (version 0.4.9) was used for visual analysis of the survival data. For data filtering, the duplicate samples were removed. The grouping method with the minimum $\mathrm{p}$ value was adopted for survival analysis. The prognostic data were provided by Liu Jianfang et al.'s article [25]. Receiver operating characteristic (ROC) curves were established by the "pROC" package (version 1.17.0.1) and visualized by the "ggplot2" package (version 3.3.3). For data filtering, duplicate samples were removed.

\section{Statistical analysis}

All statistical analyses were conducted using R (version 3.6.3) software in this study. The significance of the expression of MDKIPTN in tumor and normal tissues was analysis using the Student's t test (twotailed), the Mann-Whitney test (two-tailed), or one-way analysis of variance (ANOVA). Overall survival (OS) curves were analyzed by the log-rank test. The correlation analysis was conducted using Pearson correlation test or Spearman rank correlation test. $P<0.05$ was considered statistically significant.

\section{Results}

\section{MDK and PTN exhibited abnormal expression levels in CRC samples}

We first analyzed data from the TCGA cohort and found that $M D K$ was significantly upregulated $(P<0.0001)$, while $P T N$ was significantly downregulated $(P<0.0001)$ in $C R C$ samples versus normal tissues (Fig. 1a and 1b). Pearson correlation analysis showed no statistically significant correlation between the mRNA expression levels of PTN and MDK $(\mathrm{r}=0.050, \mathrm{P}=0.279)$, suggesting that the expression of $P T N$ and $M D K$ may be subjected to unrelated regulatory mechanisms during the occurrence and development of CRC (Fig. 2).

\section{MDK and PTN were associated with multiple biological pathways in CRC}

To further elucidate the biological functions of $M D K$ and PTN in CRC, we performed GO and KEGG signaling pathway enrichment analyses based on the LinkedOmics database. The results showed that $M D K$ was mainly enriched with immune response and inflammatory response pathways in CRC and was also involved in RNA metabolic process and cell cycle regulation (Fig. 1c and 1d). Meanwhile, PTN was mainly enriched with metabolic process and DNA replication pathways (GO pathways) in CRC (Fig. 1e and $1 \mathrm{f})$.

\section{MDK and PTN were correlated with immune functions in CRC}

Based on the LinkedOmics database, we performed GSEA on MDK and PTN in CRC. The results showed that the expression of $M D K$ was significantly positively correlated with that of genes involved in humoral immune response, adaptive immune response, $T$ cell activation, lymphocyte-mediated immunity, cell 
killing, regulation of inflammatory response, natural killer (NK) cell-mediated cytotoxicity, differentiation of Th1 and Th2 cells, intestinal immune network for IgA production, Th17 cell differentiation, inflammatory bowel disease, and autoimmune thyroid disease, and so on, in CRC ( $p<0.05$, false discovery rate $[F D R]<0.05 ;$ Fig. 3). Furthermore, the expression of $P T N$ was significantly negatively correlated with that of genes implicated in NADH dehydrogenase complex assembly, tricarboxylic acid metabolic process, mitochondrial RNA metabolic process, protein localization to chromosome, cytoplasmic translation, DNA damage response, detection of DNA damage, pentose phosphate pathway, glyoxylate and dicarboxylate metabolisms, pyruvate metabolism, DNA replication, fructose and mannose metabolisms, citrate cycle (TCA cycle), and so on, in CRC ( $p<0.05$, FDR<0.05; Fig. 4).

\section{Associations of MDK and PTN with immune infiltration}

Since our GSEA found that MDK and PTN played important immunological roles in CRC samples, we next sought to determine the relationships between $M D K$ and PTN expression and the levels of infiltrating immune cells. Based on the TIMER database, we discovered that the expression of $M D K$ was negatively correlated with tumor purity, and positively correlated with B cell, neutrophil and dendritic cell (DC) infiltration in CRC tissues (Fig. 5a). Meanwhile, PTN expression was also negatively correlated with tumor purity, but positively correlated with $\mathrm{B}$ cell, CD8+ T cell, $C D 4+\mathrm{T}$ cell, macrophage, neutrophil and dendritic cell infiltration in CRC tissues (Fig. 5a). Based on the "GSVA" package (1.34.0 version) of R (3.6.3 version), the correlations of $M D K$ and PTN expression with the infiltration of various subtypes of aDCs (activated DCs), B cells, CD8+ T cells, cytotoxic cells, DCs, eosinophils, iDCs (immature DCs), macrophages, mast cells, neutrophils, NK cells, CD56 ${ }^{\text {bright }}$ cells [26], NK CD56 ${ }^{\text {dim }}$ cells [26], NK cells, pDCs (plasmacytoid DCs), T cells, T helper cells, Tcm (T central memory) cells, Tem (T effector memory) cells, Tfh (T follicular helper) cells, Tgd (T gamma delta) cells, Th1 cells, Th17 cells, Th2 cells, and Tregs (regulatory T cells) were comprehensively analyzed. The results (Fig. 5b, Table 1) were consistent with those obtained based on the TIMER database (Fig. 5a). We also calculated the immune score, stromal score, and ESTIMATE score for each CRC patient by using the "estimate" package (version 1.0.13) in R (version 3.6.3). MannWhitney $\mathrm{U}$ analysis revealed that the expression levels of $M D K$ and PTN were both positively correlated with immune score, stromal score, and ESTIMATE score of the CRC patients (Fig. 5c). 
Table 1

Correlation analysis between MDK and PTN expression and the infiltration of immune cells in CRC

PTN

Cell type

Cell type

aDCs

B cells

CD8 T cells

Cytotoxic cells

DCs

Eosinophils

iDCs

Macrophages

Mast cells

Neutrophils

NK CD56bright cells

NK CD56dim cells

NK cells

pDCs

T cells

T helper cells

Tcm cells

Tem cells

Tfh cells

Tgd cells

Th1 cells

Th17 cells

Th2 cells

Tregs
Correlation coefficient

(Pearson)

0.192

0.298

0.238

0.170

0.332

0.355

0.459

0.455

0.562

0.262

$-0.109$

0.096

0.454

0.197

0.205

0.222

0.153

0.268

0.405

0.378

0.292

$-0.180$

0.084

0.290
MDK

Correlation coefficient $\quad p$ value

(Pearson) (Pearson)

(Pearson)

$<0.001$

0.275

$<0.001$

$<0.001$

0.157

$<0.001$

$<0.001$

0.119

0.011

$<0.001$

0.335

$<0.001$

$<0.001$

0.248

$<0.001$

$<0.001$

0.190

$<0.001$

$<0.001$

0.328

$<0.001$

$<0.001$

0.178

$<0.001$

$<0.001$

0.199

$<0.001$

$<0.001$

0.168

$<0.001$

0.021

0.301

$<0.001$

0.041

0.242

$<0.001$

$<0.001$

0.238

$<0.001$

$<0.001$

0.084

0.074

$<0.001$

0.280

$<0.001$

$<0.001$

$-0.139$

0.003

0.001

$-0.127$

0.007

$<0.001$

0.244

$<0.001$

$<0.001$

0.214

$<0.001$

$<0.001$

0.037

0.432

$<0.001$

0.252

$<0.001$

$<0.001$

$-0.101$

0.031

0.073

$-0.097$

0.038

$<0.001$

0.300

$<0.001$ 


\section{Prognostic analysis of MDK and PTN in CRC samples}

We analyzed the overall survival (OS), disease-free survival (DSS) and progression-free interval (PFI) of CRC patients and found that low MDK expression was associated with a better prognosis in terms of OS (hazard ratio $[\mathrm{HR}]=1.58,95 \%$ confidence interval $(\mathrm{Cl})$ : 1.00-2.49; $\mathrm{p}=0.052$ ) (Fig. 6a) and DSS (HR=2.26, 95\% Cl: 1.18-4.33; $p=0.016$ ) (Fig. 6b), but was not significantly associated with the PFI of CRC patients $(\mathrm{HR}=1.34,95 \% \mathrm{Cl}$ : 0.93-1.94; $\mathrm{p}=0.116)$ (Fig. 6c). We also analyzed the associations between MDK expression and OS of CRC patients in the T1/T2 and T3/T4 stages. The results indicated that MDK expression correlated negatively with the OS of T1/T2-stage CRC patients (HR=2.99, 95\% Cl: 0.57-15.55; $\mathrm{P}$ $=0.194)$ (Fig. $6 \mathrm{~d}$ ) but positively with the OS of T3/T4-stage CRC patients (HR=1.62, 95\% Cl: 1.01-2.61; $\mathrm{p}=0.048$ ) (Fig. 6e). Meanwhile, high PTN expression was associated with a better prognosis in terms of OS (HR=0.65, 95\% Cl: 0.44-0.96; $p=0.031$ ) (Fig.e 6f), but was not significantly associated with the DSS (HR=0.66, 95\% Cl: 0.40-1.09; $p=0.103$ ) (Fig. 6g) and PFI (HR=0.85, 95\% Cl: 0.58-1.23; $p=0.378)$ (Fig. 6h) of CRC patients. We also analyzed the correlations between PTN expression and OS of CRC patients in the $\mathrm{T} 1 / \mathrm{T} 2$ and T3/T4 stages. The findings revealed that PTN expression had no significant correlation with the OS of patients with T1/T2-stage CRC (HR=0.74, 95\% Cl: 0.14-3.80; $p=0.715)$ (Fig. 6i) but had a negative correlation with the OS of patients with T3/T4-stage CRC (HR=0.67, 95\% Cl: 0.44-1.00; $p=0.052)$ (Fig. 6j). In addition, the area under the ROC curve (AUC) values for the expression of MDK and PTN were 0.682 and 0.970 , respectively, in CRC patients (Fig. 6k and 6I), indicating that the expression levels of $M D K$ and $P T N$ were closed related with the prognosis of CRC. Therefore, these two genes are potential biomarkers for CRC.

\section{Discussion}

MDK and PTN are two members of the heparin-binding growth factor family of cytokines. These two proteins are highly expressed in multiple embryonic and malignant tissues. MDK is a cysteine-rich protein with a molecular weight of $13 \mathrm{kDa}[20,27,28]$, while PTN is a secretory protein with a molecular weight of $18 \mathrm{kDa}$ [8]. Prior evidence suggests that MDK and PTN can interact with several proteins, such as anaplastic lymphoma kinase (ALK), syndecans (SDCs), receptor-type protein-tyrosine phosphatase (RPTP), low-density lipoprotein receptor related protein (LRP), integrins, neuroglycan C (NGC), and Notch [18], all of which are involved in promoting tumor growth, tumor invasion and angiogenesis $[9,29]$.

Many studies have demonstrated that both MDK and PTN are abnormally expressed in CRC tissues (Table 2). Aridome, K. et al. [27] analyzed the expression of MDK in various gastrointestinal carcinomas, including gastric cancer, liver cancer, pancreatic cancer, duodenal cancer, and esophageal cancer. They found that the mRNA and protein expression levels of MDK in CRC tissues were much higher than those in adjacent tissues. Barderas, R. et al. [20] found via stable isotope labeling by amino acids in cell culture (SILAC), a high-throughput proteomic analysis approach, that the protein level of MDK in KM12SM, a highly metastatic CRC cell line, was significantly higher than that in KM12C, a lowly metastatic CRC cell line. After the expression of MDK in KM12SM cells was knocked down by siRNA interference, the metastatic ability of the cells was significantly reduced. In addition, MDK gene had also been involved in 
a six-gene prognostic signature (including CD137L, CTSS, SOSTDC1, ZG16B, EFNA3 and MDK) for CRC [20]. It was found that the high expression of this signature was significantly correlated with a poor prognosis of CRC. The higher the expression of the signature genes, the shorter the survival and the worse the prognosis of CRC patients. However, some studies have also found that the expression of MDK gene was low in the serum of CRC patients. Kemik, O. et al. [28] found that the serum levels of albumin, MDK, adiponectin and ghrelin in esophageal, gastric, pancreatic, colon and rectal cancer patients were lower than those in the healthy control group. These findings were confirmed by another study from the same research group [30]. 
Table 2

Existing studies on MDK and PTN in CRC

\begin{tabular}{|c|c|c|c|c|}
\hline $\begin{array}{l}\text { Related } \\
\text { studies and } \\
\text { journals } \\
\text { they are } \\
\text { published } \\
\text { in }\end{array}$ & $\begin{array}{l}\text { Year of } \\
\text { publication }\end{array}$ & $\begin{array}{l}\text { Gene } \\
\text { investigated }\end{array}$ & Main findings & $\begin{array}{l}\text { Gene } \\
\text { expression } \\
\text { level }\end{array}$ \\
\hline $\begin{array}{l}\text { Mol Cell } \\
\text { Proteomics } \\
{[20]}\end{array}$ & 2013 & $M D K$ & $\begin{array}{l}\text { Knockdown of } M D K \text { caused a significant } \\
\text { decrease in the migration and invasion } \\
\text { abilities of highly metastatic cells. }\end{array}$ & High \\
\hline $\begin{array}{l}\text { Hum Exp } \\
\text { Toxicol [30] }\end{array}$ & 2012 & $M D K$ & $\begin{array}{l}\text { MDK was lowly expressed in gastric } \\
\text { cancer patients' serum. }\end{array}$ & Low \\
\hline $\begin{array}{l}\text { Int J } \\
\text { Colorectal } \\
\text { Dis [31] }\end{array}$ & 2012 & $M D K$ & $\begin{array}{l}\text { MDK was lowly expressed in colon cancer } \\
\text { tissues. }\end{array}$ & Low \\
\hline $\begin{array}{l}\text { World J } \\
\text { Surg Oncol } \\
\text { [28] }\end{array}$ & 2010 & MDK & $\begin{array}{l}\text { MDK was lowly expressed in colon cancer } \\
\text { patients' serum. }\end{array}$ & Low \\
\hline $\begin{array}{l}\text { Jpn J } \\
\text { Cancer Res } \\
\text { [27] }\end{array}$ & 1995 & $M D K$ & $\begin{array}{l}\text { The increased expression of } M D K \text { in } \\
\text { gastric carcinoma was more significant in } \\
\text { well- and moderately-differentiated } \\
\text { adenocarcinomas than in poorly- } \\
\text { differentiated adenocarcinomas and } \\
\text { signet ring cell carcinomas. }\end{array}$ & High \\
\hline $\begin{array}{l}\text { PLoS One } \\
\text { [38] }\end{array}$ & 2017 & PTN & $\begin{array}{l}\text { PTN is a secretory cytokine expressed in } \\
\text { various cancer cell lines and human } \\
\text { tumors such as colon cancer, lung cancer, } \\
\text { gastric cancer and melanoma. It plays } \\
\text { significant roles in angiogenesis, } \\
\text { metastasis, cell differentiation and cell } \\
\text { growth. }\end{array}$ & High \\
\hline $\begin{array}{l}\text { Int J } \\
\text { Colorectal } \\
\text { Dis [31] }\end{array}$ & 2012 & PTN & $\begin{array}{l}\text { High PTN expression levels are } \\
\text { accompanied by high VEGF expression } \\
\text { and are predictive of a poor prognosis in } \\
\text { CRC patients. }\end{array}$ & High \\
\hline $\begin{array}{l}\text { Recent Pat } \\
\text { Anticancer } \\
\text { Drug } \\
\text { Discov [29] }\end{array}$ & 2007 & PTN & $\begin{array}{l}\text { PTN is expressed at lower levels in CRC } \\
\text { tissues than in adjacent normal mucosae. }\end{array}$ & Low \\
\hline $\begin{array}{l}\text { Cancer Lett } \\
\text { [19] }\end{array}$ & 1999 & PTN & $\begin{array}{l}\text { PTN and PTPzeta mRNA levels were } \\
\text { decreased in CRC tissues as compared } \\
\text { with adjacent normal mucosae. }\end{array}$ & Low \\
\hline $\begin{array}{l}\text { J Natl } \\
\text { Cancer Inst } \\
\text { [39] }\end{array}$ & 1998 & PTN & $\begin{array}{l}\text { pancreatic cancer }(n=41 ; P<0.0001) \text { and } \\
\text { colon cancer }(n=65 ; P=0.0079) \text { but not in } \\
\text { patients with stomach cancer }(n=87 ; \\
P=0.42)\end{array}$ & High \\
\hline
\end{tabular}


PTN has also been implicated in CRC. According to Yamakawa, T. et al. [19] and Mikelis, C. et al. [29], the mRNA levels of PTN and protein tyrosine phosphatase zeta (PTP zeta) were decreased in CRC tissues compared with adjacent noncancerous tissues. However, another study found that the expression of PTN in CRC tissues was much higher than that in normal colorectal tissues [31]. Consistent with this study, serum levels of PTN in CRC patients were found to be significantly higher than those of healthy volunteers [31]. In the same study, PTN expression was also found to be associated with CRC prognosis and tumor node metastasis classification (TNM) stage [31]. Additionally, high levels of PTN were accompanied by high expression of vascular endothelial growth factor (VEGF) and were predictive of a poor prognosis in CRC patients [31].

According to the aforementioned existing studies on MDK and PTN, these two genes seem to have different expression patterns in CRC-most studies found that MDK was highly expressed and PTN was lowly expressed in CRC. This is consistent with the findings of our analysis of data from TCGA databasethe expression of $M D K$ in CRC tissues was significantly higher than that in adjacent normal tissues, while the expression of PTN in CRC tissues was significantly lower than that in adjacent noncancerous tissues. Although PTN and MDK share similar amino acid sequences [7] and have many common biological functions, such as those associated with neurodevelopment and tumor growth, but they have different expression patterns in CRC and play different roles in the regulation of CRC development. It is interesting that opposite expression patterns of MDK and PTNalso exist in many other tumors, such as breast cancer, lung adenocarcinoma, esophageal carcinoma, kidney renal papillary cell carcinoma, and so on. The mechanism of this phenotype has not been confirmed, but we hypothesize that the different expression patterns may be associated with different tumor environments, and MDK and PTN may have participated in different biological processes. The GSEA results showed that MDK and PTN were associated with significantly different biological pathways in CRC. MDK was mainly involved in immunerelated signaling pathways, while PTN was mainly involved in metabolic signaling pathways in CRC.

It has been reported that the PTN and $M D K$ are aberrantly expressed under many inflammatory conditions, such as acute injury [32], hypoxia [33], atherosclerosis [34], and rheumatoid arthritis [35, 36]. However, it is not yet clear how they contribute to the inflammatory environment of cancer. In this study, the correlations between MDK and PTN expression and the infiltration of immune cells in CRC were analyzed based on the TIMER database. Interestingly, we found that the expression levels of $M D K$ and PTN had significant correlations with the infiltration of different types of immune cells. For example, the infiltration of Thelper cells and Tcm cells was negatively correlated with the expression of MDK but positively correlated with the expression of PTN. The infiltration of pDCs and CD8+ T cells was positively correlated with the expression of PTN but negatively correlated with the expression of MDK. In addition, the infiltration of CD8+ T cells, CD4+ T cells and macrophages was positively correlated with the expression of PTNbut had no significant correlation with the expression of $M D K$. It is well known that CD8+ $T$ cells, CD4+ $T$ cells and macrophages can kill tumor cells. Among them, $C D 8+T$ cells are the most lethal $\mathrm{T}$ cells that can kill antigen-expression tumor cells, and are important effector cells in anti-virus infection, acute allograft rejection and tumor cell elimination. Therefore, the number of CD8+ T cells 
directly determines the body's ability of eliminating tumor cells [37]. This may explain why high PTN expression was associated with a higher survival rate of CRC patients in our study.

\section{Limitation}

Our study has several limitations. First, the exact mechanisms of action of $M D K$ and PTN in CRC remain unclear. In addition, we are not sure if immune cells are the targets or the "providers" of abnormal expression levels of $M D K$ and PTN in CRC. Due to these limitations, our study is still far from clarifying the detailed functions of $M D K$ and PTN in CRC. Despite these limitations, our study lays a foundation for exploring the mechanisms of action of MDK and PTN in CRC, providing further support for the development of novel targeted drugs.

\section{Declarations}

Acknowledgments We would acknowledge the TCGA and GEO databases for providing the platforms and contributors for uploading their meaningful datasets. We also would like to thank TopEdit (www.topeditsci.com) for English language editing of this manuscript.

Author contribution All authors read and approved the final manuscript. The main contributions of each author are as follows: The authors' contributions were as follows: YL and HLH design the experiments, and drafting the article; FQJ collect and analyze the data. All of the authors reviewed and approved the final manuscript.

Funding The work was supported by the Zhejiang Provincial Natural Science Foundation of China under Grant No. LY17H160004, and the Key Project of Science and Technology Project of Huzhou City under Grant No. 2018GZ35.

Conflict of interest The authors declare no competing interests.

\section{References}

1. Atkin W, Wooldrage K, Parkin DM, et al. Long term effects of once-only flexible sigmoidoscopy screening after 17 years of follow-up: the UK Flexible Sigmoidoscopy Screening randomised controlled trial. Lancet. 2017;389(10076):1299-311. https://doi.org/10.1016/S0140-6736(17)303963.

2. Biller LH, Schrag D. Diagnosis and Treatment of Metastatic Colorectal Cancer: A Review. JAMA. 2021;325(7):669-85. https://doi.org/10.1001/jama.2021.0106.

3. Siegel RL, Miller KD, Goding Sauer A, et al. Colorectal cancer statistics, 2020. CA Cancer J Clin. 2020;70(3):145-64. https://doi.org/10.3322/caac.21601.

4. Labianca R, Nordlinger B, Beretta GD, et al. (2010) Primary colon cancer: ESMO Clinical Practice Guidelines for diagnosis, adjuvant treatment and follow-up. Ann Oncol 21 Suppl 5:v70-77. 
https://doi.org/10.1093/annonc/mdq168.

5. van der Stok EP, Spaander MCW, Grunhagen DJ, et al. Surveillance after curative treatment for colorectal cancer. Nat Rev Clin Oncol. 2017;14(5):297-315. https://doi.org/10.1038/nrclinonc.2016.199.

6. Skarkova V, Kralova V, Vitovcova B, et al. (2019) Selected Aspects of Chemoresistance Mechanisms in Colorectal Carcinoma-A Focus on Epithelial-to-Mesenchymal Transition, Autophagy, and Apoptosis. Cells 8(3). https://doi.org/10.3390/cells8030234.

7. Christman KL, Fang Q, Kim AJ, et al. Pleiotrophin induces formation of functional neovasculature in vivo. Biochem Biophys Res Commun. 2005;332(4):1146-52. https://doi.org/10.1016/j.bbrc.2005.04.174.

8. Xu C, Zhu S, Wu M, et al. Functional receptors and intracellular signal pathways of midkine (MK) and pleiotrophin (PTN). Biol Pharm Bull. 2014;37(4):511-20. https://doi.org/10.1248/bpb.b13-00845.

9. Kadomatsu K, Muramatsu T. Midkine and pleiotrophin in neural development and cancer. Cancer Lett. 2004;204(2):127-43. https://doi.org/10.1016/S0304-3835(03)00450-6.

10. Nakagawara A, Milbrandt J, Muramatsu T, et al. Differential expression of pleiotrophin and midkine in advanced neuroblastomas. Cancer Res. 1995;55(8):1792-7.

11. Lin H, Zhou Q, Wu W, et al. Midkine Is a Potential Urinary Biomarker for Non-Invasive Detection of Bladder Cancer with Microscopic Hematuria. Onco Targets Ther. 2019;12:11765-75. https://doi.org/10.2147/OTT.S235134.

12. Shin DH, Jo JY, Kim SH, et al. (2020) Midkine Is a Potential Therapeutic Target of Tumorigenesis, Angiogenesis, and Metastasis in Non-Small Cell Lung Cancer. Cancers (Basel) 12(9). https://doi.org/10.3390/cancers12092402.

13. Jager R, List B, Knabbe $C$, et al. Serum levels of the angiogenic factor pleiotrophin in relation to disease stage in lung cancer patients. $\mathrm{Br} \mathrm{J}$ Cancer. 2002;86(6):858-63. https://doi.org/10.1038/sj.bjc.6600202.

14. Ibusuki M, Fujimori $\mathrm{H}$, Yamamoto $\mathrm{Y}$, et al. Midkine in plasma as a novel breast cancer marker. Cancer Sci. 2009;100(9):1735-9. https://doi.org/10.1111/j.1349-7006.2009.01233.x.

15. Chang Y, Zuka M, Perez-Pinera $P$, et al. Secretion of pleiotrophin stimulates breast cancer progression through remodeling of the tumor microenvironment. Proc Natl Acad Sci U S A. 2007;104(26):1088893.

16. Jee YH, Sadowski SM, Celi FS, et al. Increased Pleiotrophin Concentrations in Papillary Thyroid Cancer. PLoS One. 2016;11(2):e0149383. https://doi.org/10.1371/journal.pone.0149383.

17. Choi YW, Kim YH, Lee J, et al. Strong immunoexpression of midkine is associated with multiple lymph node metastases in BRAFV600E papillary thyroid carcinoma. Hum Pathol. 2015;46(10):155765. https://doi.org/10.1016/j.humpath.2015.06.018.

18. Sorrelle N, Dominguez ATA, Brekken RA. From top to bottom: midkine and pleiotrophin as emerging players in immune regulation. J Leukoc Biol. 2017;102(2):277-86. https://doi.org/10.1189/jlb.3MR1116-475R. 
19. Yamakawa T, Kurosawa N, Kadomatsu K, et al. Levels of expression of pleiotrophin and protein tyrosine phosphatase zeta are decreased in human colorectal cancers. Cancer Lett. 1999;135(1):916. https://doi.org/10.1016/s0304-3835(98)00275-4.

20. Barderas R, Mendes $M$, Torres $S$, et al. In-depth characterization of the secretome of colorectal cancer metastatic cells identifies key proteins in cell adhesion, migration, and invasion. Mol Cell Proteomics. 2013;12(6):1602-20. https://doi.org/10.1074/mcp.M112.022848.

21. Vasaikar SV, Straub P, Wang J, et al. LinkedOmics: analyzing multi-omics data within and across 32 cancer types. Nucleic Acids Res. 2018;46(D1):D956-63. https://doi.org/10.1093/nar/gkx1090.

22. Li T, Fan J, Wang B, et al. TIMER: A Web Server for Comprehensive Analysis of Tumor-Infiltrating Immune Cells. Cancer research. 2017;77(21):e108-10. https://doi.org/10.1158/0008-5472.CAN-170307.

23. Yoshihara K, Shahmoradgoli M, Martínez E, et al. Inferring tumour purity and stromal and immune cell admixture from expression data. Nature communications. 2013;4:2612. https://doi.org/10.1038/ncomms3612.

24. Yoshihara K, Shahmoradgoli M, Martinez E, et al. Inferring tumour purity and stromal and immune cell admixture from expression data. Nat Commun. 2013;4:2612. https://doi.org/10.1038/ncomms3612.

25. Liu J, Lichtenberg T, Hoadley KA, et al. (2018) An Integrated TCGA Pan-Cancer Clinical Data Resource to Drive High-Quality Survival Outcome Analytics Cell 173(2):400-16 e411. https://doi.org/10.1016/j.cell.2018.02.052.

26. Michel T, Poli A, Cuapio A, et al. Human CD56bright NK Cells: An Update. J Immunol. 2016;196(7):2923-31. https://doi.org/10.4049/jimmunol.1502570.

27. Aridome K, Tsutsui J, Takao S, et al. Increased midkine gene expression in human gastrointestinal cancers. Jpn J Cancer Res. 1995;86(7):655-61. https://doi.org/10.1111/j.13497006.1995.tb02449.x.

28. Kemik O, Sumer A, Kemik AS, et al. The relationship among acute-phase response proteins, cytokines and hormones in cachectic patients with colon cancer. World J Surg Oncol. 2010;8:85. https://doi.org/10.1186/1477-7819-8-85.

29. Mikelis C, Koutsioumpa M, Papadimitriou E. Pleiotrophin as a possible new target for angiogenesisrelated diseases and cancer. Recent Pat Anticancer Drug Discov. 2007;2(2):175-86.

30. Kemik O, Kemik AS, Begenik $\mathrm{H}$, et al. The relationship among acute-phase responce proteins, cytokines, and hormones in various gastrointestinal cancer types patients with cachectic. Hum Exp Toxicol. 2012;31(2):117-25. https://doi.org/10.1177/0960327111417271.

31. Kong Y, Bai P-S, Nan K-J, et al. Pleiotrophin is a potential colorectal cancer prognostic factor that promotes VEGF expression and induces angiogenesis in colorectal cancer. Int $\mathrm{J}$ Colorectal Dis. 2012;27(3):287-98. https://doi.org/10.1007/s00384-011-1344-z.

32. Sato W, Kadomatsu K, Yuzawa Y, et al. Midkine is involved in neutrophil infiltration into the tubulointerstitium in ischemic renal injury. J Immunol. 2001;167(6):3463-9. 
33. Weckbach LT, Groesser L, Borgolte J, et al. Midkine acts as proangiogenic cytokine in hypoxiainduced angiogenesis. Am J Physiol Heart Circ Physiol. 2012;303(4):H429-38. https://doi.org/10.1152/ajpheart.00934.2011.

34. Li F, Tian F, Wang L, et al. Pleiotrophin (PTN) is expressed in vascularized human atherosclerotic plaques: IFN-\{gamma\}/JAK/STAT1 signaling is critical for the expression of PTN in macrophages. FASEB J. 2010;24(3):810-22. https://doi.org/10.1096/fj.09-140780.

35. Maruyama $\mathrm{K}$, Muramatsu $\mathrm{H}$, Ishiguro $\mathrm{N}$, et al. Midkine, a heparin-binding growth factor, is fundamentally involved in the pathogenesis of rheumatoid arthritis. Arthritis Rheum. 2004;50(5):1420-9.

36. Pufe $T$, Bartscher $M$, Petersen $W$, et al. Expression of pleiotrophin, an embryonic growth and differentiation factor, in rheumatoid arthritis. Arthritis Rheum. 2003;48(3):660-7.

37. Jansen CS, Prokhnevska N, Master VA, et al. An intra-tumoral niche maintains and differentiates stem-like CD8 T cells. Nature. 2019;576(7787):465-70. https://doi.org/10.1038/s41586-019-1836-5.

38. Zha L, He L, Xie W, et al. Therapeutic silence of pleiotrophin by targeted delivery of siRNA and its effect on the inhibition of tumor growth and metastasis. PloS one. 2017;12(5):e0177964. https://doi.org/10.1371/journal.pone.0177964.

39. Souttou B, Juhl H, Hackenbruck J, et al. Relationship between serum concentrations of the growth factor pleiotrophin and pleiotrophin-positive tumors. J Natl Cancer Inst. 1998;90(19):1468-73.

\section{Figures}


a

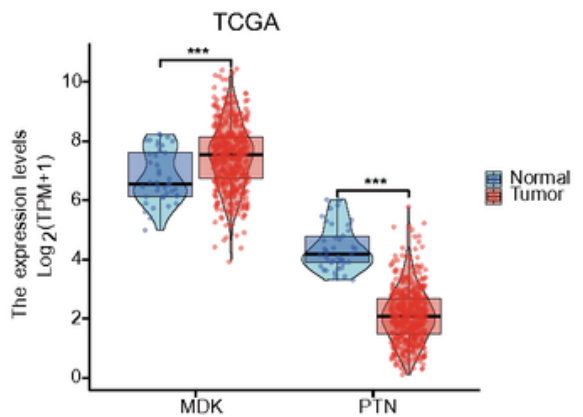

C

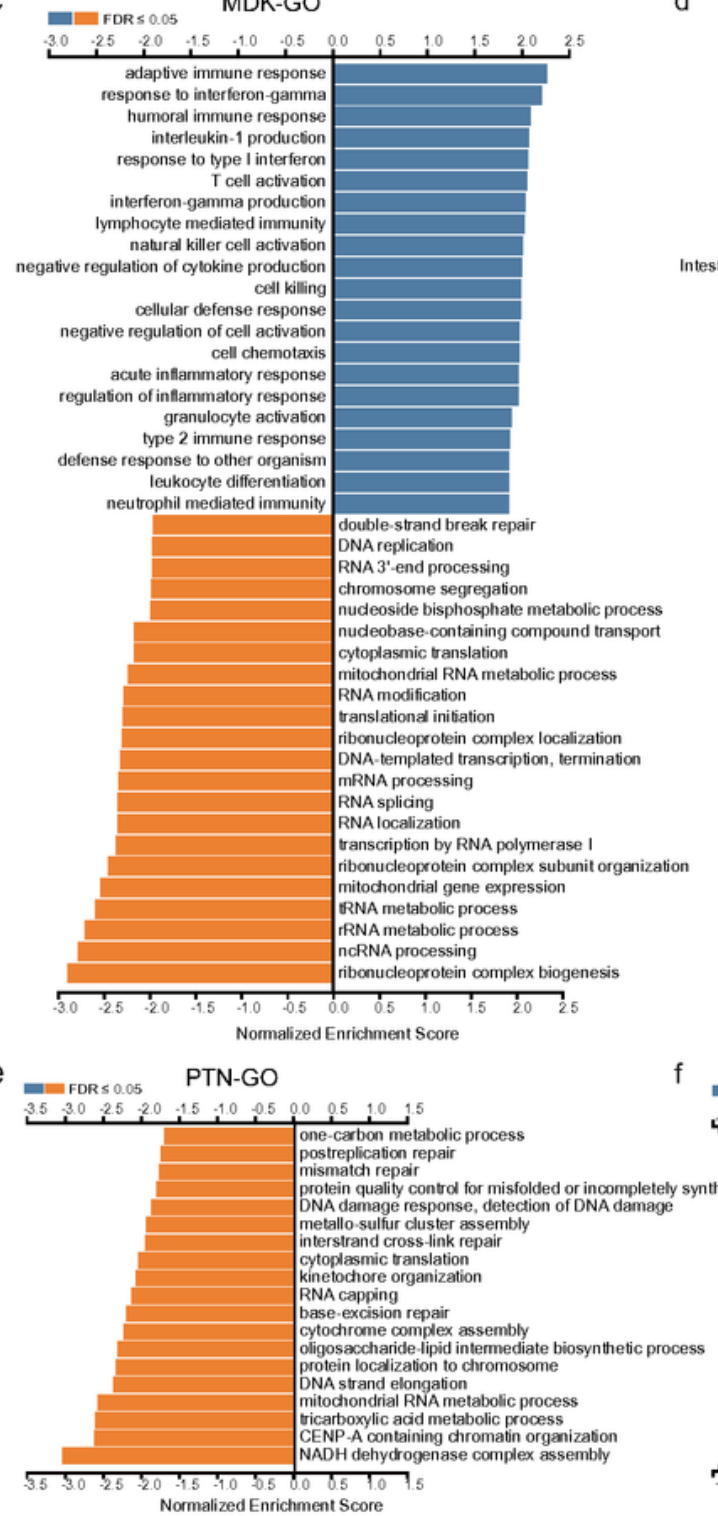

b

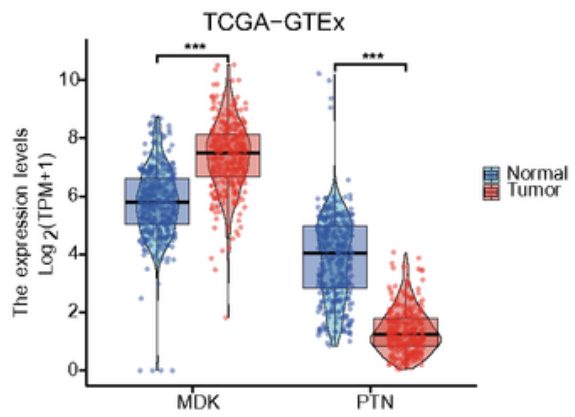

d

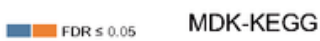

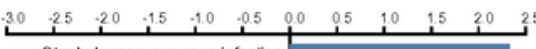

Staphylococcus aureus infection

Graft-versus.host disease
Rheumatoid arthrifs

Rheumatoid arthritis

Cell adhesion molecules (CAMs)

Natural killer cell medated cytotoxicity

Antigen processing and presentation

Th1 and Th2 cell differentiatio

estinal immune network for IgA production

Inflammatory bowel disease (IBD)

Allograft rejection

Cytokine-cytokine receptrasome

Systemic lupus erythematosus

Osteoclast differentiatio

Tuberculosis
Toxoplasmosis

Toxoplasmosis

Type I diabetes mellitus
Epstein-Bar virus infection

Steroid biosynthesis

Terpenoid backbone biosynthesis

RNA polymerase

Fatty acid metabolism

Cell cyde

Vitamin digestion and absorption

Selenocompound metabolism

Cysteine and methionine metabolism

Carbon metabolism

Fatty acid biosynthesis

Fanconi anemia pathway

One carbon pool by folate

nRNA surveillance pathw

yruvate metabolism

Basal transcription factors

Citrate cyde (TCA cycle)

Propanoate met

Spliceosome

Ribosome

Aminoacyl-RRA biosynthesis

Ribosome biogenesis in eukaryotes

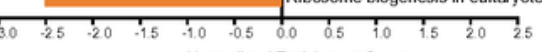

Normalized Enrichment Score
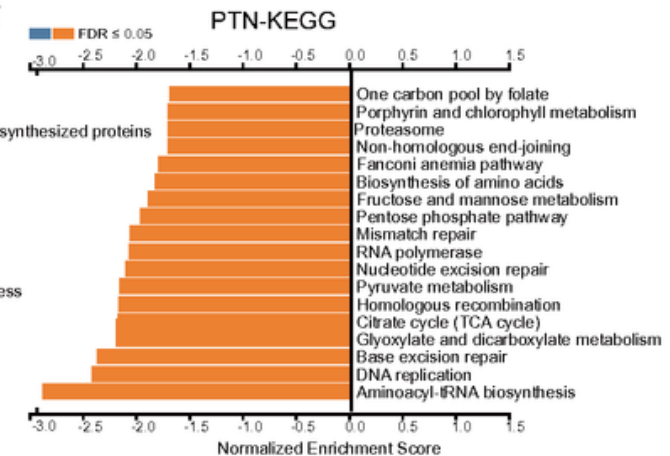

Figure 1

Expression levels and functions of $M D K$ and PTN in CRC. Expression levels of $M D K$ and PTN in CRC according to TCGA (a) and TCGA-GTEx (b) databases. GO analysis of MDK (c) and PTN (e) and KEGG analysis of $M D K(\mathrm{~d})$ and $P T N(\mathrm{f})$ based on the LinkedOmics database. 


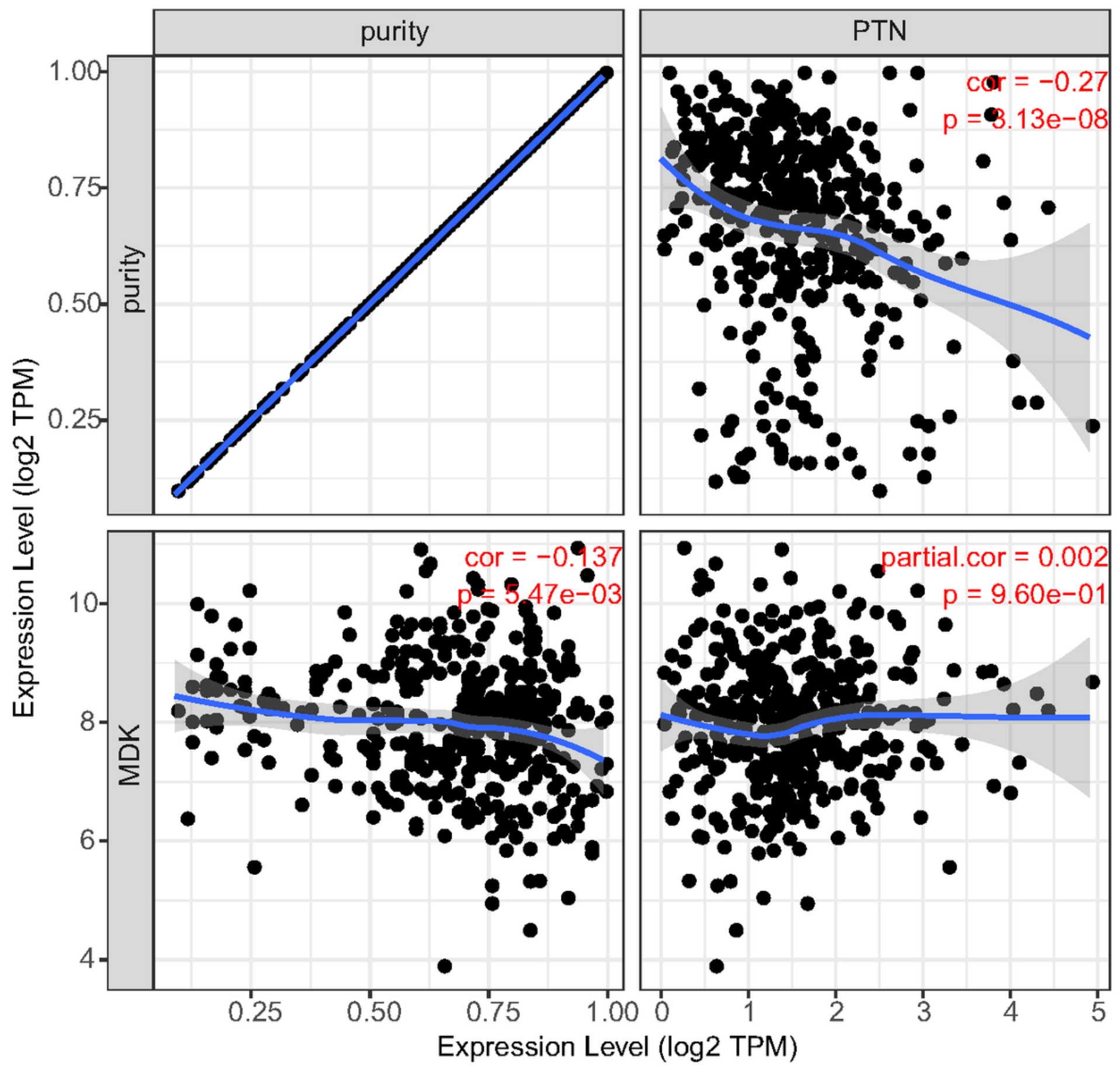

Figure 2

Correlations between expression levels of $M D K$ and PTN and cancer purity. 

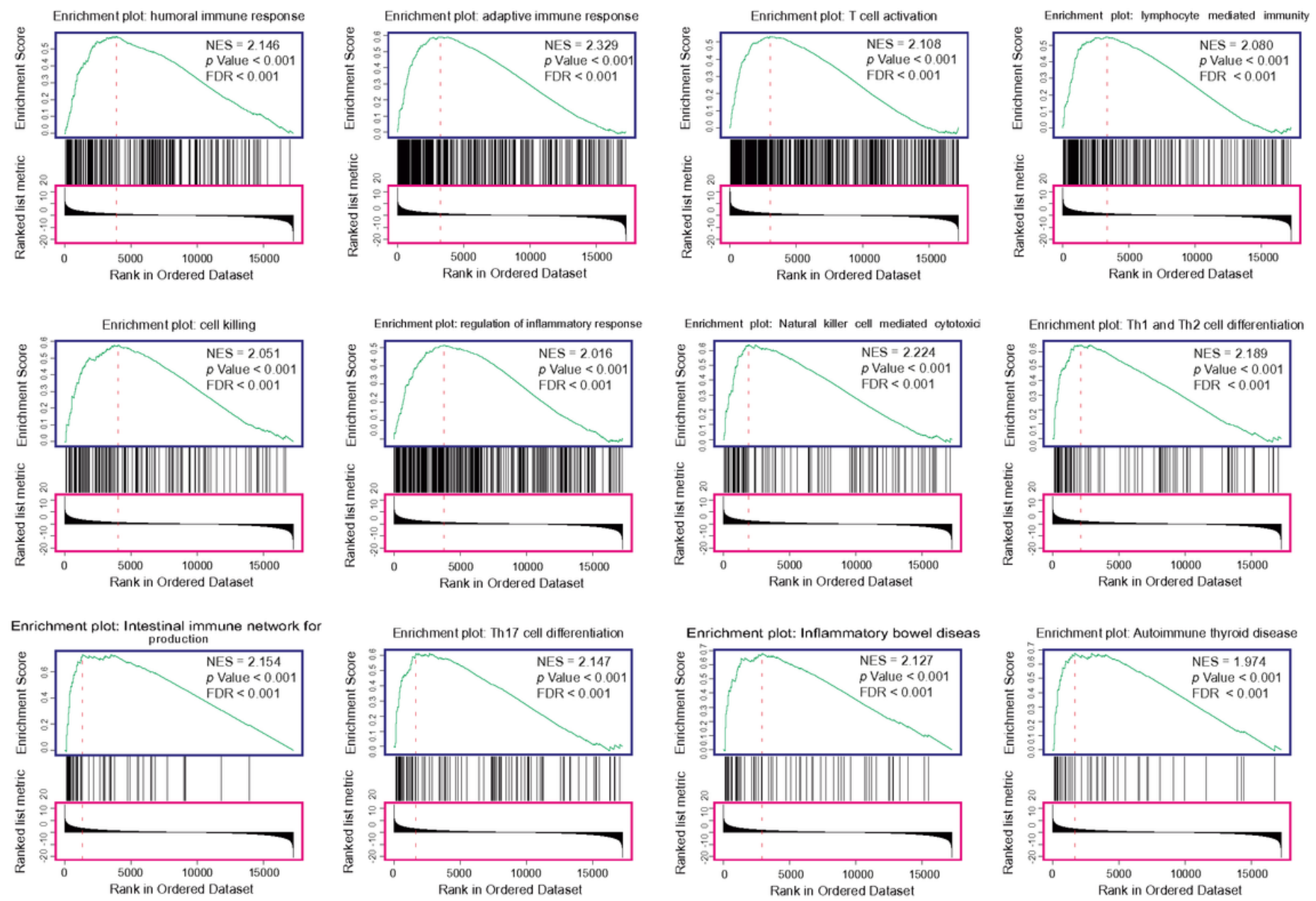

Figure 3

GSEA of $M D K$ in CRC. 

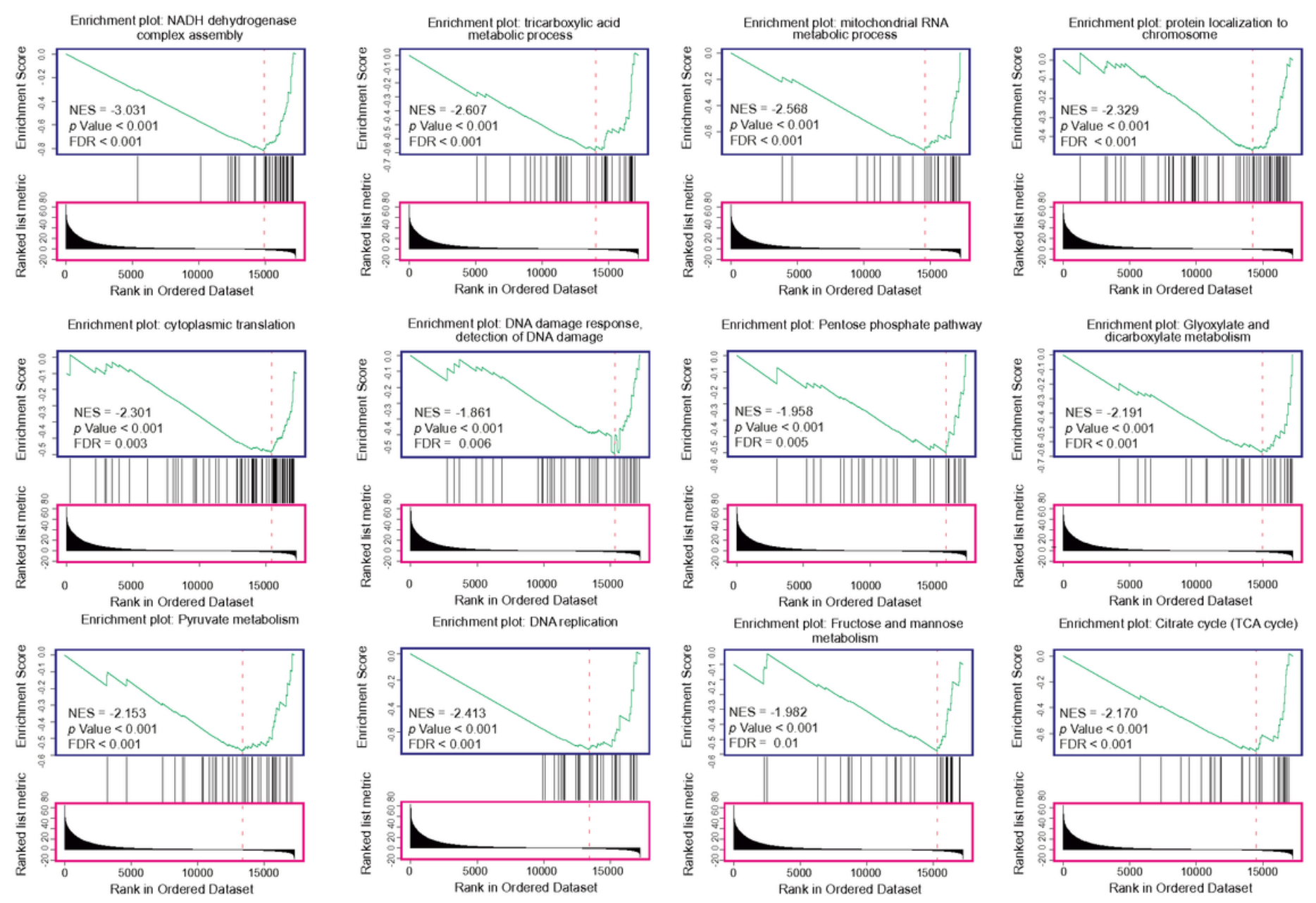

Figure 4

GSEA of PTN in CRC. 

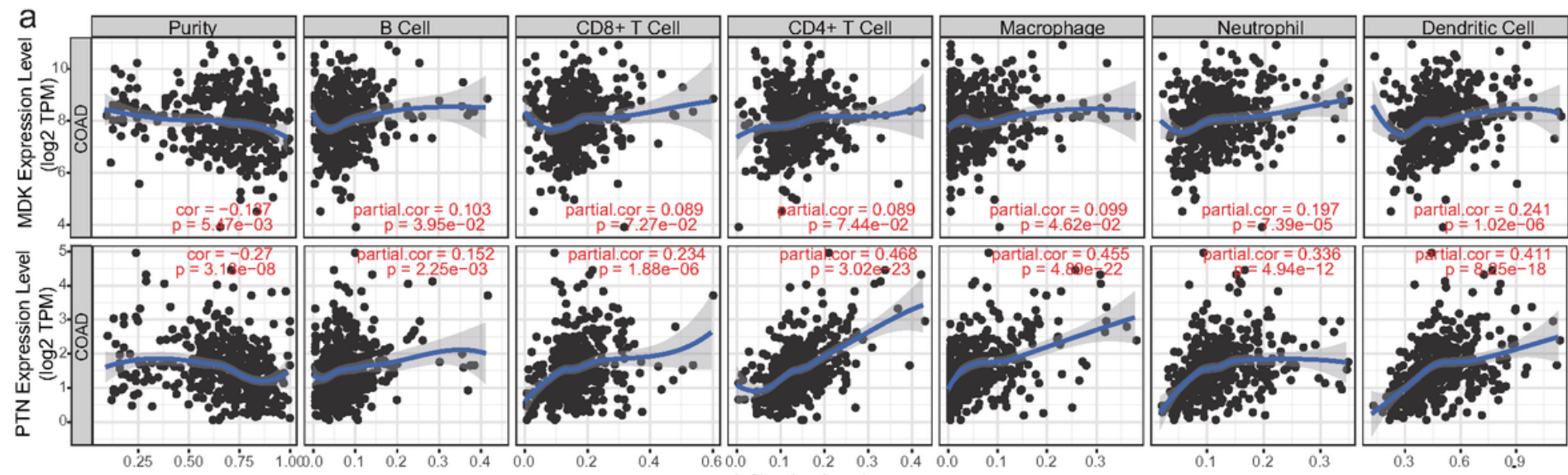

b
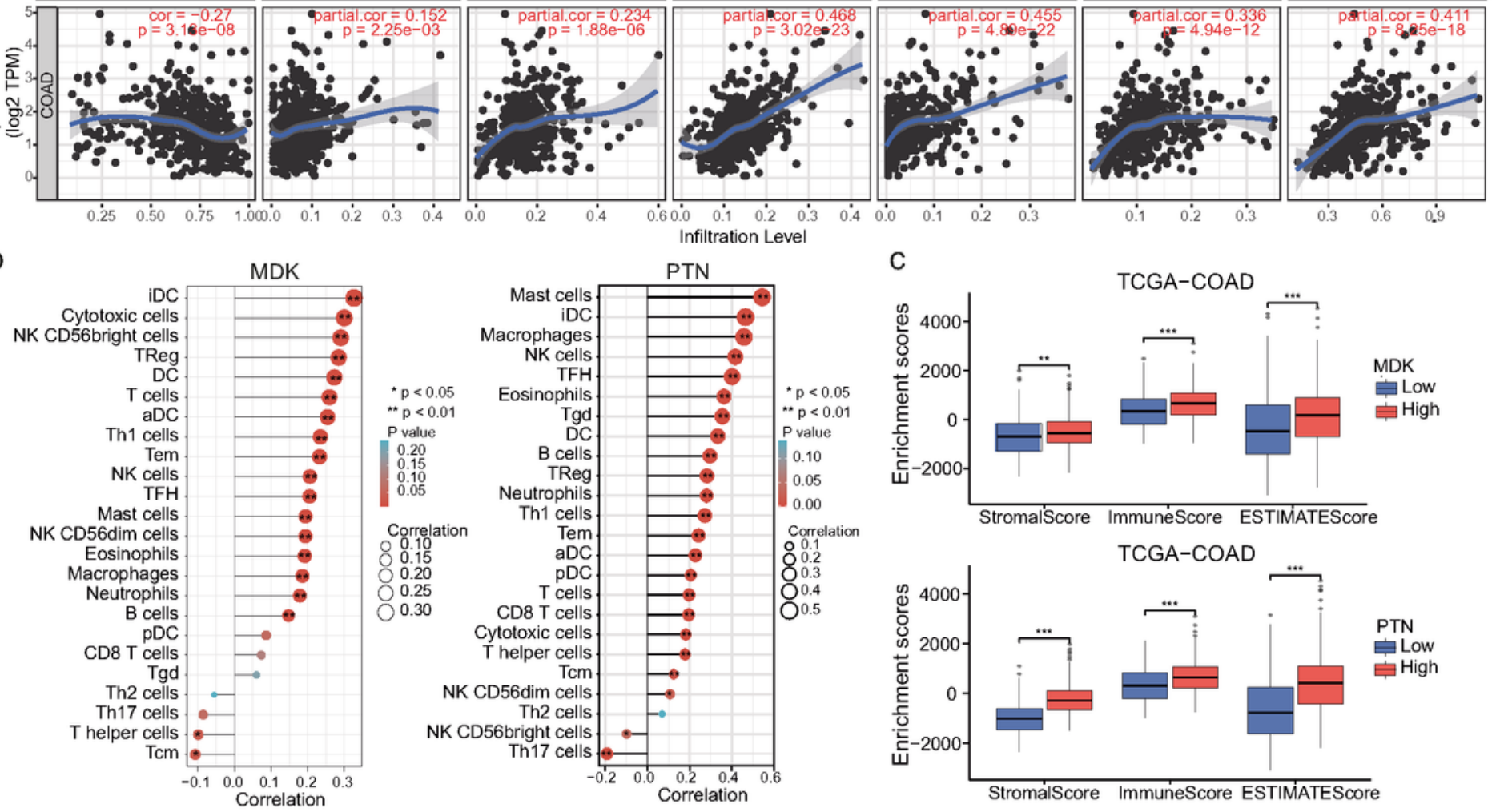

C
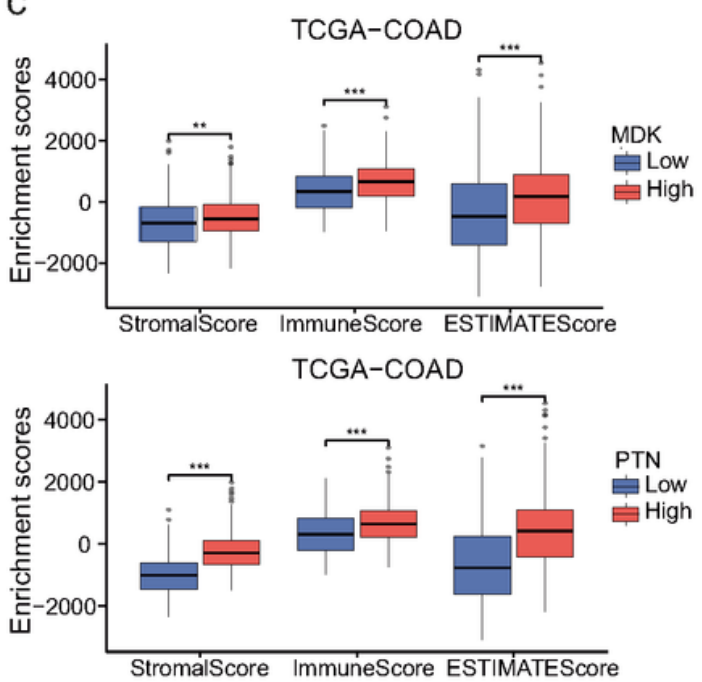

Figure 5

a, Correlations between expression levels of $M D K$ and PTN and tumor purity, B cell infiltration, neutrophil infiltration and dendritic cell infiltration in CRC. b, Correlations between expression levels of MDK and PTN and the infiltration of various subtypes of immune cells. c, Correlations between expression levels of MDK and PTN and immune score, stromal score, and ESTIMATE score for each CRC patient. 

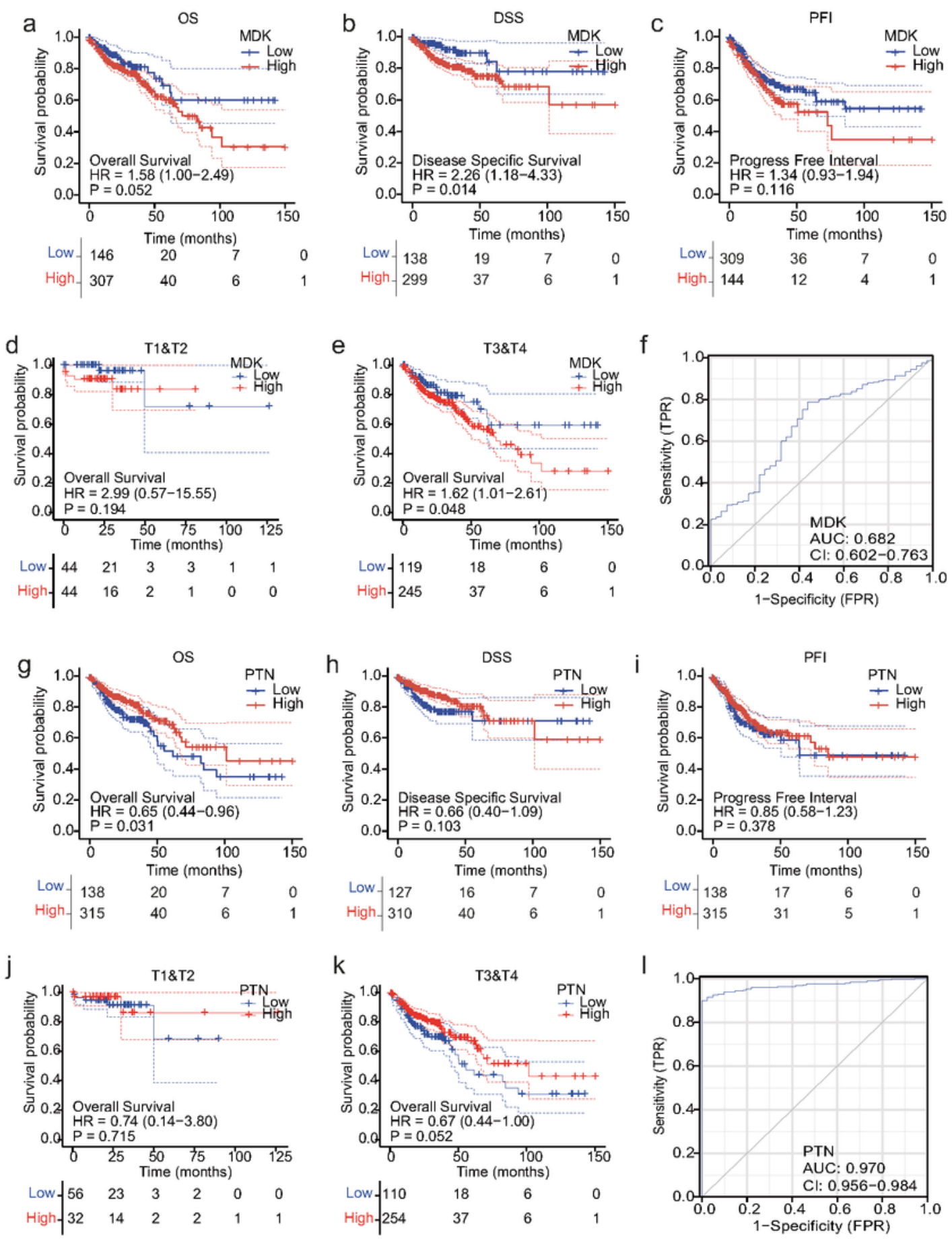

\section{Figure 6}

Prognostic value of MDK and PTN in CRC. The OS (a), DSS (b), and PFI (c) of CRC patients with different levels of MDK expression. The OS of T1/T2-stage CRC (d) and T3/T4-stage CRC (e) patients with different levels of MDK expression. The AUC of MDK expression in CRC patients (f). The OS (g), DSS (h), and PFI (i) of CRC patients with different levels of PTN expression. The OS of T1/T2-stage CRC (j) and 
T3/T4-stage CRC (k) patients with different levels of PTN expression. The AUC of PTN expression in CRC patients (l). 